\title{
Increase in frequency of publication: a new era for PHCR\&D
}

With the publication of this issue of PHCR\&D, Volume 16.1, the Journal is entering a new era. Publication of the Journal increases from four issues a year to six issues from the beginning of 2015. When we launched the Journal in 2000, we hoped that others would appreciate that the Journal was aimed at providing a forum for publication of papers that cross the primary health care (PHC) research, development, teaching and practice interfaces. Increase in the frequency of publication indicates that people in the world-wide PHC community have recognised this purpose. In recent years, there has been a significant growth in the number of high-quality papers submitted to the Journal and this increase has led directly to the decision by the Journal publisher, Cambridge University Press, to increase the frequency of publication of the Journal.

When the Journal was launched, the purpose was to publish both research and development papers. The back issues of the Journal provide a rich depository of papers concerned with research methods, the contribution of different professional groups to PHC (including general practitioners, nurses, pharmacists, professions allied to medicine among others), research concerned with health issues (including health of older people, diabetes, mental health, teenage health), research concerned with education of communities and practitioners and papers concerned with the development of PHC. Uniquely in 2000, we set out to publish papers providing research evidence of how change is achieved in PHC practice. In the intervening years, Implementation Science, as this area has become to be known, has grown across many disciplines enabling growth in the understanding of mechanisms to improve services and individual practice. This depository also provides evidence and ideas for future research and scholarship. Implementation Science uses diverse methods and approaches to further the understanding of complex health care issues; the trend in the Journal has been towards publishing papers that use a range of methodologies to address real world problems. There have been many examples over the past 15 years of research and development papers that inform PHC practitioners and researchers about health care matters ranging from breastfeeding in Finland (Tarkka, 2001) to End of Life Care for people with dementia (Raymond et al., 2014), real life cycle topics that are meaningful both for those working in primary care and those living in our global communities.

In 2013, PHCR\&D introduced the Cochrane Summary papers. These have provided the opportunity to precis the evidence in Cochrane Reviews and to enable researchers and practitioners to have readily available to them the key messages from Cochrane Reviews, which is also an important aspect of Implementation Science. These have covered topics such as childhood immunisation, postpartum depression and smoking cessation. The reviews are among the most downloaded papers and have been a popular addition to the Journal. We plan to continue these in collaboration with the Cochrane Nursing Group and to ensure access to high-quality evidence.

In July 2014, the European Union Expert Panel on Effective Ways of Investing in Health had their opinion on the definition of primary care adopted:

'The provision of universally accessible, person-centred, comprehensive health and community services provided by a team of professionals accountable for addressing a large majority of personal health needs. These services are delivered in a sustained partnership with patients and informal caregivers, in the context of family and community, and play a central role in the overall coordination and continuity of people'scare'.

(European Commission, 2014, p. 18)

This definition, accepted across the European Union, ensures a framework for thinking critically about primary care/PHC, the questions that it raises for research across all types of conditions and settings, which have in common the need for accessible, person-centred and comprehensive care. As editors of the Journal, we wish to 


\section{Rosamund Bryar and Sally Kendall}

encourage new and exciting research papers that challenge the assumptions about primary care/ PHC. As the Journal grows and develops over the next decade, our aim, as editors in chief, is therefore to continue to publish the highest standards of research and development work in primary care and papers that are concerned as well with the wider field of PHC. There will be a continued emphasis in the Journal on health and well-being in community and population-based settings and multidisciplinarity but with some fresh approaches to methodology and implementation. We invite you to contribute to our on-going goal of becoming the leading journal for PHC research and development.

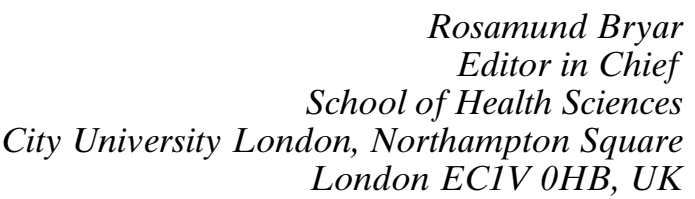

Rosamund Bryar Editor in Chief on, Northampton Square London ECIV OHB, UK
Sally Kendall

Editor in Chief

Centre for Research in Primary and Community

Care (CRIPACC)

University of Hertfordshire, College Lane

Hatfield, Herts AL1 1SW, UK

\section{References}

European Commission. 2014: Definition of primary care opinion adopted by health expert panel. Director General Health and Consumers. Retrieved 2 December 2014 from http://ec.europa.eu/health/expert_panel/opinions/docs/001_ definitionprimarycare_en.pdf

Raymond, M., Warner, A., Davies, N., Nicholas, N., Manthorpe, J. and Iliffe, S. 2014: Palliative and end of life care for people with dementia: lessons for clinical commissioners. Primary Health Care Research \& Development 15, 406-17.

Tarkka, M.-T. 2001: Guidance on breastfeeding by public health nurses has an impact on competence of first-time mothers. Primary Health Care Research and Development 2, 80-87. 